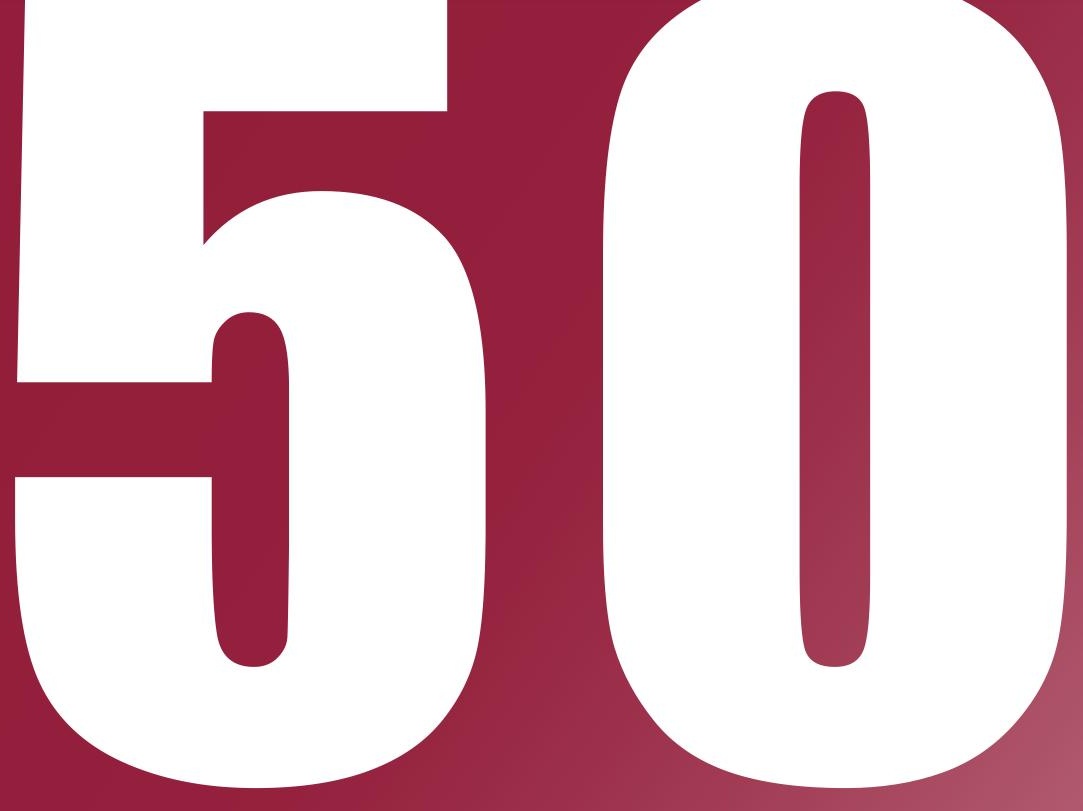

ÁMBITOS

REVISTA

INTERNACIONAL

DE

COMUNICACIÓN

$\mathbf{N}^{\circ} \mathbf{5 0}$

EDICIÓN OTOÑO

2020

ISSN: 1139-1979

E-ISSN: 1988-5733

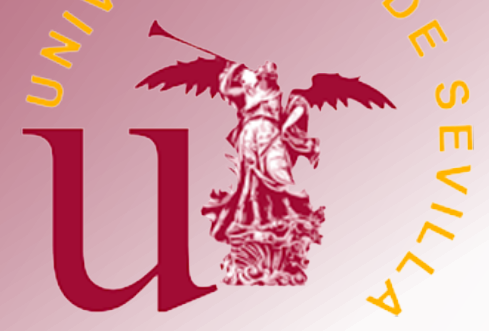




\section{INDICE}

EDITORIAL EDITORIAL

Presentación del Monográfico. Comunicación y juventud. Nuevos medios, representación, recepción y participación en contenidos de entretenimiento e información

Presentation of the monograph. Communication and youth. New media, representation, reception and participation in entertainment and information content.

Juan Francisco Gutiérrez Lozano, Francisco Javier Ruiz del Olmo

7-9

\section{MONOGRÁFICO MONOGRAPH}

El consumo audiovisual de la Generación Z. El predominio del vídeo online sobre la televisión tradicional The audiovisual consumption of Generation $Z$. The predominance of online video over traditional television

La imagen de los jóvenes en las estrategias transmedia de las series de televisión: el caso de Skam España en Instagram

The image of young people in transmedia strategies of TV series: the case of Skam España on Instagram

Sergio Jesús Villén Higueras, Francisco Javier Ruiz del Olmo

La penetración de Netflix en el público español ¿cuestiona el modelo televisivo tradicional?

Does the arrival of Netflix in the Spanish audience challenge the traditional television model?

Javier Bustos Díaz

49-61

Generación Z y consumo de información política: entre la televisión y los nuevos formatos mediáticos Generation $Z$ and consumption of political information: between television and new media formats

Ruth Gómez de Travesedo Rojas, Marta Gil Ramírez

$62-79$

Actualidad y comedia: El éxito de El Intermedio y La Resistencia entre los espectadores jóvenes

Information and comedy: The Success of El Intermedio and La Resistencia for young audiences

Inmaculada Concepción Aguilera García

80-95 
El efecto socializador de articular un espacio de comunidad virtual en el ecosistema del aula

The socializing effect of creating a virtual community space in the classroom environment

Lorea Ariadna Ruiz Gómez, Mónica Hinojosa Becerra, Francisco Javier Ruiz San Miguel

Juventude ciberativista e educação: reflexões sobre um jeito hacker de ser

Cyber activity youth and Education: reflections on a hacker way of being

Carla Azevedo de Aragão, Pietro Matheus Bompet Fontoura Alves, Karina Moreira Menezes

La risa grotesca en la obra de Bob Dylan: análisis de la Bacanal y el Festín Carnavalesco en la trilogía folk-rock eléctrica

Grotesque Laughter in Bob Dylan's Work: Analysis of The Bacchanal and the Carnival Feast in the Electric Folk-rock Trilogy

Jesús Albarrán Ligero

El papel del intertexto en el videojuego. Una partida, mil y una historias

Intertext in video games. A game, thousand and one stories

Rocío Serna-Rodrigo

145-158

\section{ÁmBitos PERSONALES PERSONAL ÁmBITOS}

El auge de Twitch: nuevas ofertas audiovisuales y cambios del consumo televisivo entre la audiencia juvenil The rise of Twitch: New audiovisual offers and the transformation of Television consumption among young audiences

\section{ARTÍCULOS ARTICLES}

La recepción de los medios de comunicación para residentes rusos en la Costa del Sol

Mass media reception by Russian residents on the Costa del Sol

Natalia Meléndez Malavé, José Carlos Pozo García

176-192

La calidad periodística en la cobertura de terremotos: Caso Ecuador

Journalistic quality in earthquake coverage: The case of Ecuador

Juan Pablo Arrobo-Agila, Mendoza María, Ignacio Aguaded

La credibilidad publicitaria en la nueva esfera mediática: los universitarios y los medios

Advertising credibility in the new media sphere: university students and the media 
Arco iris en medios brasileños: percepciones sociales sobre una campaña publicitaria LGBTQIA+

Rainbow in Brazilian media: social perceptions of an LGBTQIA+ advertising campaign

Leonardo Ferreira Batista, José Geraldo de Araújo Ramalho Filho

224-239

Rituales y comunicación política: la toma de posesión de los presidentes autonómicos españoles

Rituals and political communication: the Spanish autonomous Presidents inauguration

Ricardo Domínguez García

240-258

RESEÑAS REVIEWS

Una mirada diferente para hacer periodismo

A different look for making journalism

Guillermo Paredes-Otero

259-261

Periodismo y tecnología: una simbiosis continua

Journalism and technology: a continuous symbiosis

Luisa Graciela Aramburú Moncada

262-265

Una propuesta metodológica para impartir la asignatura de redacción periodística

Journalism and technology: a continuous symbiosis

Noelia Ruiz-Alba

266-268 


\title{
La recepción de los medios de comunicación para residentes rusos en la Costa del Sol ${ }^{1}$
}

\author{
Mass media reception by Russian residents on the Costa del Sol
}

\author{
Natalia Meléndez Malavé \\ Universidad de Málaga | Calle de León Tolstoi, s/n, 29010, Málaga \\ | España | http://orcid.org/0000-0003-0835-3997 | natalia@uma.es \\ José Carlos Pozo García \\ Universidad de Málaga | Calle de León Tolstoi, s/n, 29010, Málaga \\ | España | https://orcid.org/0000-0002-2692-6173| josecarlospozo@uma.es
}

Fechas | Recepción: 14/04/2020 | Aceptación: 16/07/2020 | Publicación final: 15/10/2020

\begin{abstract}
Resumen
Este trabajo se propone conocer a la comunidad rusa asentada en la Costa del Sol desde una perspectiva sociocomunicativa, dado su destacable aumento poblacional en España en los últimos 15 años, parejo a la aparición casi simultánea de medios de comunicación locales dirigidos a estos residentes en su idioma. Se pretende profundizar en el estudio de los hábitos y preferencias mediáticas de este colectivo inmigrante, así como sus impresiones sobre los medios en ruso y sus percepciones sobre la contribución de estos últimos al grado de integración en la sociedad española. Para ello, se realizó tanto un registro detallado de la docena de medios para la población rusa en la zona, como un estudio de recepción basado en técnicas cuantitativas (encuesta) como cualitativas (grupo de discusión). Los resultados de esta indagación constatan que para el $89 \%$ de los encuestados dichos medios locales en
\end{abstract}

\begin{abstract}
This paper examines the Russian community on the Costa del Sol from a sociocommunicative perspective. It explores their significant population increase in Spain over the past 15 years and the almost simultaneous appearance of locally produced Russian language mass media targeted at them. The study looks into the habits and media preferences of these immigrants and investigates their views on these media and their contribution to the integration of this population into the Spanish society. For this purpose, a register of a dozen media in Russian language in this area was compiled and a study of media reception using quantitative (survey) and qualitative techniques (focus group) undertaken. The results show that, according to $89 \%$ of respondents, this local Russian language media provides them with better knowledge of Spain in general, and $80 \%$ believe that it helps them gain a greater
\end{abstract}


ruso les aportan un mayor conocimiento del territorio de destino. Además, un $80 \%$ manifiesta que les ayuda a conocer mejor la sociedad española.

Palabras clave: medios de comunicación, Keywords: mass media, foreign residents, residentes extranjeros, prensa extranjera, foreign press, Russian press in Spain, Russian prensa rusa en España, rusos en España, in Spain, Russian diaspora. diáspora rusa.

\section{INTRODUCCIÓN}

La presente investigación ha sido desarrollada dentro de un proyecto más amplio centrado a nivel general en el estudio de la comunidad de residentes europeos en la Costa del Sol española y El Algarve portugués, a través de nueve nacionalidades representativas -entre ellas, la rusacon el objetivo de conocer qué medios de comunicación para extranjeros consumen, cuáles son las motivaciones para su uso y cuáles son sus percepciones sobre estos medios. En última instancia, esta investigación tiene la finalidad de indagar en el proceso de integración de estos residentes europeos y si en ello influyen, de algún modo, los medios de comunicación para extranjeros.

Dado que los resultados de la investigación general antes mencionada presentan especificidades según las nacionalidades, este trabajo dirige sus miras a analizar en concreto el caso de los ciudadanos rusos que residen en la Costa del Sol. Una comunidad que ha experimentado un llamativo incremento en este territorio en las últimas décadas en España, tal y como apunta Barrera-Fernández (2014): «en 2014 había 224.000 residentes cuyos orígenes eran alguna de las repúblicas de la antigua Unión Soviética, de los cuales 64.653 eran rusos. Si comparamos esta cifra con la de 1997 (3.500 en ciudadanos), el número de residentes se ha incrementado en más de 18 veces en 17 años» ${ }^{2}$.

Parejo a este aumento de población se manifiesta el auge que han tenido los medios de comunicación en lengua rusa en los últimos años, que en la Costa del Sol se han incrementado de solo uno en 2005 (López Romero, p. 2005) a doce según nuestro censo actual elaborado para esta investigación en 2018.

En consonancia pues con los objetivos generales del proyecto, se pretende extraer datos de interés para el mejor conocimiento de los públicos a los que se dirigen estos medios, desde una óptica sociocomunicativa, para profundizar en el estudio de la comunidad rusa que habita en la Costa del Sol y cuáles son sus hábitos y preferencias mediáticas, así como sus impresiones sobre estos medios tan particulares, que se elaboran localmente, pero en su idioma. Finalmente, esta investigación se propone también conocer el grado de integración en la sociedad española de los rusos que residen en esta zona de España y la contribución que los medios estudiados puedan suponer en este aspecto.

Para el desarrollo de esta investigación, como se verá más adelante en el apartado metodológico, una de las tareas fundamentales consistió en la búsqueda de antecedentes 
bibliográficos sobre medios de comunicación y comunidades de extranjeros. Siendo este ya de por sí un ámbito limitado y relativamente reciente en cuanto al interés científico, pero que goza de una producción destacable si hablamos de estudios conjuntos o de determinadas nacionalidades -basten como ejemplo para el territorio de la Costa del Sol los trabajos de García Galindo et al. (1998, 2004, 2018, 2019, 2020), juzgamos que en el caso concreto del colectivo de ciudadanos rusos residentes en España quedan aún lagunas por cubrir, entre las que modestamente el presente estudio puede suponer una aportación novedosa.

Así pues, si nos ceñimos a la comunidad rusa, existen pocas investigaciones centradas en los residentes asentados en España. Alguna pone de manifiesto la incidencia económica de este colectivo en la costa, en este caso alicantina, como la tesis doctoral de Prokopenko (2014). Relacionado con este aspecto encontramos el ya mencionado artículo de Barrera-Fernández (2014) sobre el mercado inmobiliario ruso en la Costa del Sol. Pero sobre el ámbito concreto que nos ocupa, relacionando a este colectivo con los medios de comunicación, la aportación que más se aproxima a esta cuestión es el trabajo de Pozo García (2019) acerca del panorama mediático en lengua rusa en la Costa del Sol, que realiza un recorrido por la situación actual de la oferta mediática para rusohablantes en la zona.

Precisamente sobre el mismo grupo, pero centrado en el conjunto del territorio español y ya publicado fuera de España, encontramos otra de las grandes aportaciones al estudio mediático de esta comunidad, a cargo de Ershov y Gadzhieva (2018), en el que establecen la evolución histórica de las publicaciones para hispanoparlantes en España: «En 1995, se lanzó el primer periódico en ruso en España, Новая Земля (traducción "Nueva Tierra"). La publicación tenía una periodicidad bimensual y estaba destinada principalmente a turistas de Rusia y los estados postsoviéticos, que vacacionaban en los centros turísticos de Andalucía. En los años siguientes, con el creciente número de comunidades de habla rusa en España, aparecieron dos semanarios: Комсомольская правда в Испании ("Komsomolskaya Pravda en España") у MK-Новости Испании, "MК-Noticias de España»³ (Ershov y Gadzhieva, 2018, p. 98).

El mismo estudio señala cómo pronto se amplían los territorios de acogida de residentes rusos donde estas comunidades empiezan a contar con medios de comunicación propios: «... en la década de 2000 en las zonas turísticas de España, donde también viven muchos inmigrantes de Rusia y los países de la antigua Unión Soviética, aparecieron publicaciones periódicas y medios electrónicos en ruso, principalmente con fines informativos, publicitarios y de entretenimiento: el periódico Вести Майорка ("Vesti Mallorca”, cuya traducción sería “Noticias de Mallorca”), Русский журнал ("Revista rusa” de Barcelona), la revista Linda (Costa del Sol), revistas bilingües como ImpulsPlus (Murcia) y Beautiful People (Marbella)» (Ershov y Gadzhieva, 2018, p. 98).

Como también señalan estos autores, en los últimos años han aparecido medios importantes como la edición rusa en el verano de 2013 de Diario Sur, el principal periódico de la Costa del Sol, que ya contaba con versiones en inglés y alemán. Asimismo, en el ámbito radiofónico, a finales de 2011 en Alicante comenzó a transmitir Radio rusa y en 2013 se creó la estación de radio Russian Radio Marbella (Costa del Sol), que organizó el festival de cine ruso en Marbella en 2016. 
Hay otros artículos sobre la diáspora rusa en otros lugares del mundo, algunos de ellos abordando la cuestión de la prensa destinada a estos públicos, pero sobre todo para analizarla desde el punto de vista lingüístico. Así, Shovgenin y Shovgenina (2015) tratan el problema del funcionamiento de la lengua rusa en dos periódicos para rusohablantes publicados en territorio alemán. También Perotto (2014) analiza singularidades lingüísticas entre parientes emigrantes rusos y familias ítalo-rusas bilingües residentes en Italia. En el caso español, existe una tesis vinculada a estas cuestiones (Tereshchuk, 2017) que se centra en el estudio de la asimilación lingüística de los inmigrantes rusos en la provincia de Barcelona, analizando el contacto lingüístico del ruso y el español basándose en entrevistas realizadas a miembros de la diáspora rusohablante en la zona.

Resulta interesante asimismo citar un trabajo algo más inclasificable, pero sin duda sugerente a cargo de Andy Byford (2012) que examina el nuevo rol de "los rusos fuera de Rusia» en los asuntos exteriores, con particular interés en la reciente estrategia del gobierno ruso de desarrollar una red global de asociaciones en la diáspora, especialmente de carácter cultural, para emplearlas como instrumento para las relaciones internacionales. Sobre ellas preguntamos también a nuestros informantes, como veremos más adelante.

Relacionado con esto último, otro grupo importante de investigaciones sobre la comunidad rusa en la diáspora corresponde a aquellos estudios dedicados a explicar los motivos de este fenómeno migratorio, fuera ya de su incidencia comunicativa. Así, existen trabajos que exploran las raíces de los últimos grandes movimientos de población rusa por diversas zonas del mundo tras la caída del bloque soviético: es el caso de la tesis $A$ history of the Soviet diaspora in the post-cold war era (1989-2015): Case studies of Russian-Speakers in South Korea, Cambodia, and The Netherlands (Harmon-Donovan, 2015). En una línea parecida, pero centrada en territorio estadounidense, se sitúa el artículo "Historiography of the Russian Diaspora in the USA", (Evgenii 2015), que analiza la percepción de cuatro oleadas de emigración rusa en EE.UU.

A todo lo expuesto se añaden como complemento de interés algunas ideas al respecto de las causas de parte de la distribución de esta diáspora rusa en la era post guerra fría por países mediterráneos como las manifestadas en el ya citado trabajo de Ershov y Gadzhieva que resumen muy bien la situación: "En la década de 1990, después del colapso de la URSS, la migración fluye desde Rusia y otros estados postsoviéticos hacia Occidente, incluyendo la región mediterránea -a Italia, España, Portugal-. Junto con las oportunidades económicas de los inmigrantes de habla rusa atrajo las condiciones climáticas de estos países, su brillante cultura, amabilidad y apertura de la población local» (Ershov y Gadzhieva, 2018, p. 96). Esta explicación entronca con algunas de las aportaciones de los informantes del presente estudio en encuestas y grupos de discusión, como veremos más abajo.

\section{METOdOLOGÍA}

A nivel metodológico, este estudio ha constado de dos fases bien diferenciadas. La primera fase ha sido netamente cuantitativa y ha consistido en la elaboración de tres bases de datos: una sobre la bibliografía del objeto de estudio, que es aún escasa en cuanto a investigaciones 
académicas, como se ha evidenciado anteriormente, pero que se ha completado también con la recopilación de artículos de prensa española dedicados a la comunidad rusa y sus hábitos de vida y consumo en España; una segunda base de datos, que incluía los contactos rusos más relevantes en la Costa del Sol (consulado, asociaciones, grupos de ciudadanos y medios de comunicación) y que han servido de enlace con los informantes encuestados en la segunda fase; y una tercera base de datos que ha registrado los medios de comunicación localizados en el idioma ruso en la Costa del Sol.

La segunda fase de esta investigación ha combinado un instrumento cuantitativo -a través de una encuesta a residentes rusos mayores de 18 años que pasan más de seis meses en la Costa del Sol desde hace dos o más años- con un instrumento cualitativo, mediante un grupo de discusión, con el objetivo de profundizar en las preguntas planteadas en la encuesta.

Como se ha indicado previamente, este trabajo forma parte de un proyecto nacional de investigación mediática en el sur de España y Portugal que considera las nueve nacionalidades europeas con mayor representación de medios de comunicación en ambos territorios. A partir del número de residentes de esas nacionalidades extranjeras en la Costa del Sol -datos extraídos del INE (Instituto Nacional de Estadística)- en 2017, se hizo el cálculo proporcional para asignar el número concreto de encuestas para cada nacionalidad. De 800 encuestas realizadas en total para la investigación general, la mayor proporción correspondió a los británicos (51,7\% de la muestra), que es la comunidad de residentes europeos más numerosa; a continuación, a residentes alemanes (10,5\%); el tercer lugar lo ocupan precisamente los residentes rusos (6,9\%); y el resto pertenece a las demás nacionalidades.

La encuesta se tradujo al idioma ruso para facilitar la comprensión de todas las preguntas formuladas y se ofreció a los informantes la posibilidad de realizarla en papel o bien online (mediante un formulario de Google). Constaba de cinco grandes bloques y 41 preguntas, referidas a la mudanza a España, relaciones sociales, integración en la sociedad española, el consumo que hacen de los medios de comunicación extranjeros y el consumo que hacen de los medios producidos concretamente en la Costa del Sol. En su mayoría, las preguntas de la encuesta eran cerradas (se ofrecían varias opciones para elegir una sola), pero también había otra tipología de preguntas semiabiertas (podían responder con sus impresiones además de elegir una opción) y algunas totalmente abiertas para expresar libremente sus opiniones sobre diversos temas.

Por último, se desarrolló un grupo de discusión que reunió a cinco participantes rusos con los mismos requisitos exigidos a los encuestados sobre mayoría de edad, tiempo de permanencia al año en la Costa del Sol y años vividos en España con el propósito de profundizar, interpretar y valorar los resultados cuantitativos obtenidos de la encuesta. Siguió una serie de pautas generales (Suárez Ortega, 2005, p. 21; Lunt y Livingstone, 1996) para esta técnica conversacional de recogida de información cualitativa, como el establecimiento de objetivos preliminares, el diseño previo, el procedimiento de desarrollo y el análisis de las dos horas que se grabaron, así como la transcripción de las impresiones, opiniones, acuerdos y desacuerdos de los intervinientes rusos en el grupo de discusión. 


\section{RESULTADOS}

\subsection{La oferta mediática local para la comunidad rusa en la Costa del Sol}

A partir de la creación de la base de datos de medios referida en el apartado anterior, se ha podido establecer un censo de doce medios destinados a los ciudadanos rusos en el período de 2016 a 2018. Cuantitativamente hay uno más con respecto al registro de medios en ruso en la Costa del Sol de octubre de 2015 (Pozo García, 2019), que contabilizó once. No obstante, hay que subrayar que el registro no es completamente exhaustivo. Se tiene constancia de la existencia de algunas emisoras de radio que emiten desde internet para los residentes y turistas rusos de la Costa del Sol, pero carecen de registro oficial como medios de comunicación en España y, en muchos casos, ni siquiera tienen datos de contacto.

Tabla 1

Tipología de medios en lengua rusa en la Costa del Sol

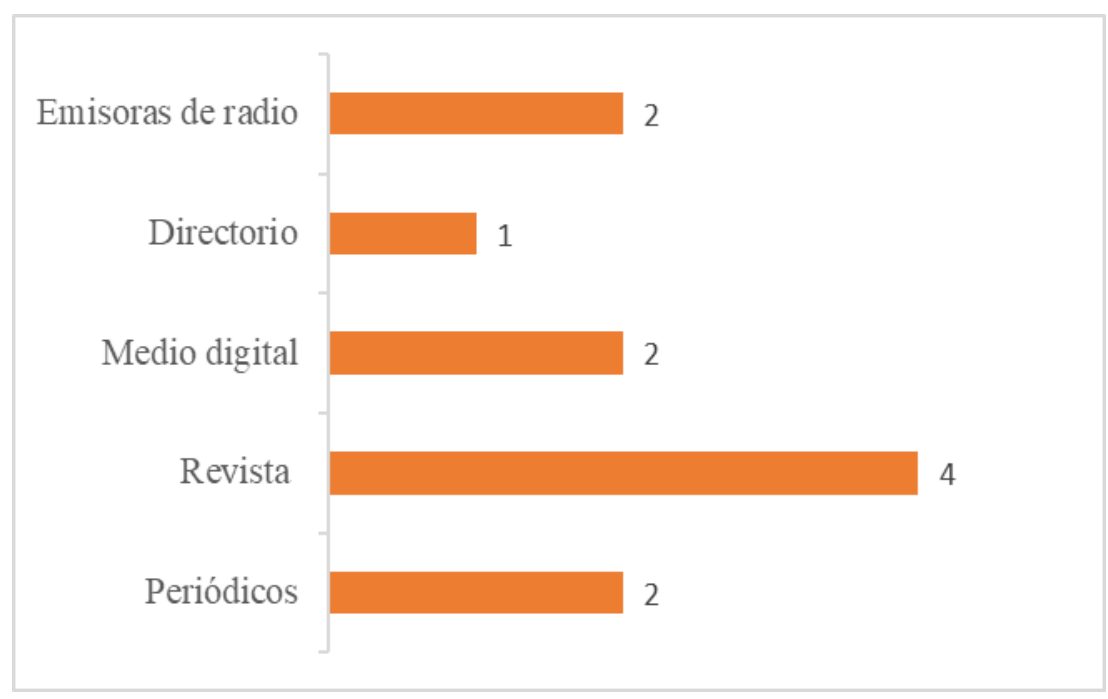

Fuente: elaboración propia.

Como muestra la tabla 1, las revistas constituyen la tipología de medios de comunicación más frecuente. Con contenidos en lengua rusa, las revistas editadas en la Costa del Sol son La vida Linda, Mecmо встречи ("Mesto vstrechii», cuya traducción es "Punto de encuentro"), Tout y Social People Marbella Magazine. A continuación, le siguen los periódicos, las emisoras de radio y los medios digitales, con dos medios para cada una de esas tipologías.

Del análisis de los contenidos que publican las cuatro revistas en ruso se extrae que su público objetivo es de alto poder adquisitivo, puesto que los contenidos versan principalmente sobre estilo de vida, lujo y eventos en la Costa del Sol. Las secciones que coinciden en tres revistas ( $L a$ vida Linda, Место встречи у Tout) son: belleza y salud, motor, gastronomía gourmet, moda y viajes. Por su parte, Social People Marbella Magazine se centra en informar sobre los diferentes eventos (campeonatos deportivos, exposiciones de arte, conciertos, etc.) que tienen lugar en Marbella y a los que acuden políticos, empresarios, cantantes, personajes de la prensa del corazón, etc. 
Los dos periódicos recogidos en este censo de medios en ruso son МК-Новости Испании («MKNoticias España») у Sur на русском ("Sur en ruso»), cuya periodicidad en ambos casos es semanal. МК-Новости Испании еs el medio más veterano que se produce en lengua rusa en la Costa del Sol. Su primer número apareció en 2000 y pertenece al grupo editorial Comunicaciones rusas en España, que también publica La vida Linda, entre otros medios. Recopila semanalmente noticias importantes acontecidas en España y las secciones fijas de economía, salud, sociedad y ciencia. Además, casi un tercio de sus páginas están dedicadas a la sección de anuncios clasificados.

Por su parte, Sur на русском comenzó a editarse en 2013. Pertenece a Prensa Malagueña S.A., dentro del grupo de comunicación Vocento, que también edita el diario Sur y la cabecera en inglés Sur in English, nacida en 1984 y de enorme relevancia como publicación para extranjeros de referencia. Las noticias que se publican en este periódico son fundamentalmente traducciones del español al ruso de las aparecidas en el diario Sur y versan sobre la actualidad informativa de Málaga y su provincia. Las páginas finales de Sur на русском son anuncios destinados a la comunidad rusohablante de la Costa del Sol.

Los dos medios digitales son Russian Spain y I-Marbella, mientras que las dos emisoras de radio registradas son RusRadio Marbella у Smile FM. Por último, Берег Солнца (su traducción al español es "Costa del Sol") es el único caso de directorio turístico y se edita una vez al año.

De estos doce medios localizados, siete son en lengua rusa y, por tanto, su público objetivo es prioritariamente rusohablante, incluyendo no solo a los ciudadanos de Rusia, sino también a otras nacionalidades que tienen ese idioma como oficial en sus países, como Bielorrusia o Kazajistán, u otras comunidades de países de la antigua Unión Soviética que tradicionalmente hablan ruso, como ucranianos, moldavos o armenios. Existe un solo medio bilingüe, Tout, el cual se edita en ruso y en inglés; y otros tres son trilingües (la emisora de radio Smile FM, la revista Social People Marbella Magazine y un medio digital I-Marbella), que se producen en español, inglés y ruso.

\subsection{Marbella, epicentro migratorio ruso}

Además de actualizar el censo de medios para conocer la oferta existente, una parte fundamental en esta investigación fue el abordaje de las características sociodemográficas más significativas de la comunidad rusa que reside en la Costa del Sol. Se trata de datos descriptivos que pueden servir para trazar un perfil de los ciudadanos de esta nacionalidad como audiencia potencial de los medios de comunicación producidos localmente en lengua rusa.

Según las encuestas realizadas, la edad media de los ciudadanos rusos es de 35 a 40 años, dato que contrasta con la concepción que tenemos de residente extranjero jubilado que busca esencialmente una mejor calidad de vida en la tercera edad. De hecho, en la muestra estudiada solo se encuentra un $2 \%$ de jubilados rusos, mientras que un $50 \%$ está compuesto por trabajadores asalariados, un $21 \%$ tiene un trabajo eventual y un $13 \%$ son desempleados. El resto se completa con estudiantes, autónomos y dueños de un negocio. Esto se ajusta a las características generales de la población rusa en la zona, muy joven en comparación con la de británicos y alemanes, que está compuesta básicamente por jubilados. Para los rusos solo el 7\% 
tiene más de 65 años. Estas cifras contrastan sustancialmente con las de otras nacionalidades: por ejemplo, la población residente británica cuenta con 47.193 ciudadanos empadronados en 2019, de los cuales el 40,9\% tiene 65 años o más (19.338 ciudadanos). Por su parte, los ciudadanos alemanes residentes en la provincia de Málaga en 2019 son 9.500, de los cuales la mayor proporción se sitúa entre los 65 años o más. En total, son 3.599, es decir, el 37,8\% ${ }^{4}$.

Respecto al género, resultó mucho más complicado localizar a hombres rusos que a mujeres de esa nacionalidad en la Costa del Sol para esta investigación: el 93\% de las participantes son mujeres. Una cifra que concuerda también con el carácter eminentemente femenino que ha tenido la emigración rusa en España en los últimos 20 años, como así se constata en el padrón de habitantes de 2018. De los 73.930 ciudadanos rusos censados en España ese año, 49.649 son mujeres, esto es, el $63 \%{ }^{5}$. De acuerdo al padrón, la provincia de Málaga es prácticamente un calco de la distribución por géneros que se da en el total de España. En 2018 la cifra es de 6.545 rusos empadronados en esta provincia, de los cuales el $65 \%$ está compuesto por mujeres.

Los encuestados rusos de este estudio residen mayoritariamente (72\%) en tres municipios de la Costa del Sol (por orden, Marbella, Málaga y Fuengirola). Según el padrón municipal de habitantes extranjeros de $2017^{6}$, las dos localidades con más ciudadanos rusos residentes son, efectivamente, Marbella (1.799 personas) y Málaga (867), seguidas por Mijas (847) y Estepona (757). De estos datos se puede inferir, pues, que la comunidad rusa de la Costa del Sol tiene Marbella como su principal epicentro poblacional.

En cuanto al año de llegada a España con la intención de residir, más de la mitad de los encuestados rusos (58\%) comenzó a vivir en el país entre 2005 y 2015. A su vez, se trata mayoritariamente de residentes permanentes, puesto que el $84 \%$ declara que reside todo el año y solo el $9 \%$ vive entre tres y seis meses en España a lo largo del año. Es clarificador a este respecto que el $89 \%$ de los rusos de este estudio afirma estar empadronado en el ayuntamiento de la localidad en la que reside. En referencia a la propiedad o no de su vivienda, prácticamente la mitad de los ciudadanos rusos vive en casa propia en la Costa del Sol y la otra mitad vive en un alojamiento alquilado.

Estos datos van asimismo en consonancia con el perfil que traza Daniel Barrera-Fernández (2014), en su caso sobre inversores en el mercado inmobiliario, pero perfectamente extrapolables a la población en general, así como a nuestros informantes: «Actualmente Rusia es el tercer país inversor en el mercado inmobiliario español (8,79\%). El perfil principal de inversores es de una pareja de clase alta, de entre 30 a 45 años, que busca una casa amplia con vistas al mar. Se trata, en su mayoría, de empresarios y directores generales de empresas. Sin embargo, en los últimos años ha aumentado también el número de familias de clase media que adquieren apartamentos».

Las tres principales razones que los residentes de la comunidad rusa enumeran para haber venido a vivir a España son el clima (25\%), la calidad de vida (19\%) y por considerarlo un sitio seguro para vivir (17\%). En una amplísima mayoría (91\%), los encuestados rusos afirman querer quedarse en España por ahora; o lo que es lo mismo, muy pocos son los que declaran su intención de cambiar de lugar de residencia. Un factor que podría ser determinante para la 
permanencia o no es tener a la familia cerca. Hay un $65 \%$ de rusos que indica que tiene familiares viviendo en España.

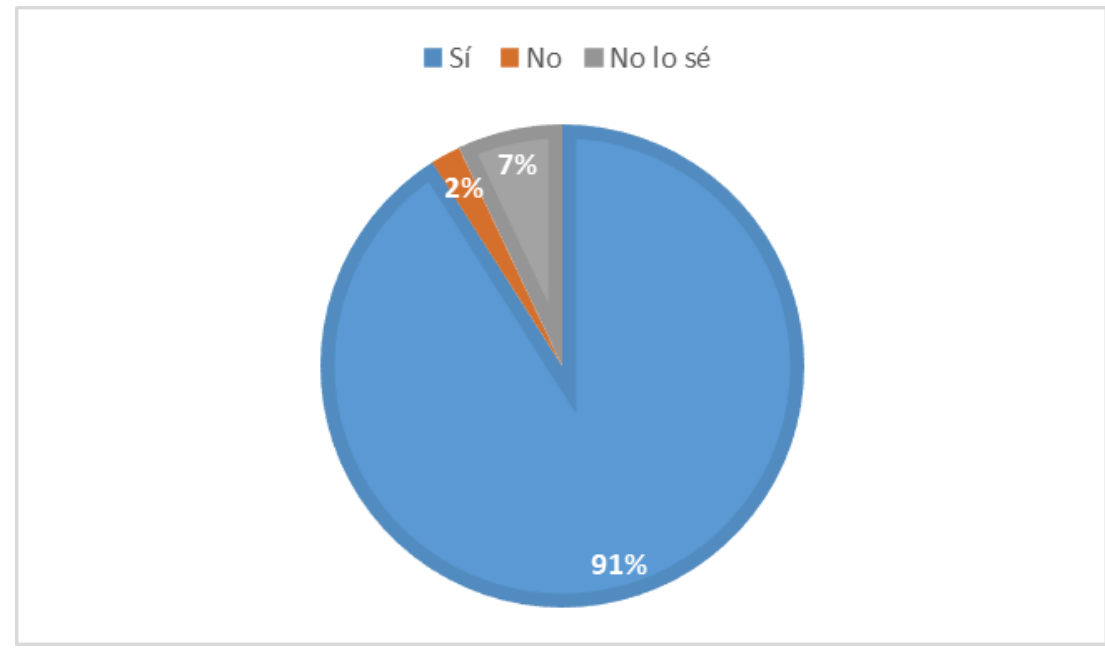

Figura 1. Resultados totales a la pregunta «¿Pretende quedarse en España por ahora?» Fuente: elaboración propia.

En los grupos de discusión los ciudadanos rusos participantes coinciden en que, con respecto a Rusia, la calidad de vida es mejor en España, y en especial en la Costa del Sol.

"La vida es más relajada, aquí se vive para vivir y no para trabajar y la gente es mucho más abierta y amable. Te sientes mejor en general (...) Me gustó esta zona en concreto, porque aquí te sientes más integrada, porque hay otros extranjeros. Si tú vas al interior de España te sientes como un outsider, como alguien de fuera y ya no estás tan cómodo. Pero en la Costa del Sol como hay otros extranjeros, es como más internacional y te sientes mejor». (Mujer, traductora, 10 años viviendo en España)

\subsection{Barreras lingüísticas y asociacionismo ruso}

Precisamente en nuestra investigación nos planteábamos también qué factores podían influir más a la hora de facilitar la integración de los rusos residentes en la Costa del Sol. Uno de ellos es el conocimiento del idioma español. En el estudio se tomaron en cuenta las cuatro destrezas lingüísticas del Marco común europeo de referencia para las lenguas (MCER) del Consejo de Europa: comprensión auditiva, expresión oral, comprensión lectora y expresión escrita. Entre los ciudadanos rusos encuestados, un 55\% afirma que habla con fluidez, un $42 \%$ que escribe con fluidez, un $49 \%$ que entiende con fluidez y un $51 \%$ que lee con fluidez. Hay que destacar que no hay ningún encuestado ruso que solo hable algunas palabras en español, esto es, que su nivel sea básico. En concreto, en la expresión oral en español el restante $45 \%$ incluye a aquellos que aseveran que su nivel de español es «bastante decente» o "se las pueden arreglar», esto es, niveles intermedios y elementales. A este conocimiento bueno o suficiente del idioma español 
habría que vincular que la mayoría de los rusos encuestados (76\%) manifiesta haber asistido alguna vez a clases de español.

En cuanto a las relaciones sociales, nos interesaba saber también si los rusos pertenecen a asociaciones o clubes como modo de integrarse en la vida comunitaria de esta zona de España. Una de las vertientes de nuestra investigación matriz fue indagar acerca de cuáles son las asociaciones de ciudadanos extranjeros en la Costa del Sol que hay registradas oficialmente. En concreto, de ciudadanos rusos se identificó una (Asociación Rusa Costa del Sol). En todo caso, son pocos los rusos encuestados que se unen a asociaciones formales (de rusos o de otras nacionalidades). Cuando lo hacen, afirman participar más en grupos informales, sobre todo, deportivos como Royal Tennis Club, Marbella C.F., etc. Hay que subrayar que en los últimos años han surgido también variados grupos de rusos en las redes sociales en España en general y en la Costa del Sol en particular, en especial en Facebook, como Círculo Ruso de Marbella, Nash Dom (asociación de rusoparlantes), Marbella y Costa de Sol rusas, Rusos en España, etc. No obstante, nuestra investigación no pretendía categorizar estos grupos en redes sociales, entre otros motivos, porque no son entidades reconocidas oficialmente, incluyen a residentes y no residentes de diferentes nacionalidades rusohablantes (bielorrusos, ucranianos, armenios, moldavos, etc.) o no tienen una estructura organizativa muy definida. Si bien es cierto que los informantes en los grupos de discusión citaron estas redes sociales informales como método de adquisición de información casi al nivel de los medios de comunicación, lo cual no deja de ser un resultado significativo.

Finalmente, tanto del estudio cuantitativo a través de la encuesta como del cualitativo mediante el grupo de discusión, se extrae que los rusos no tienen problemas para entablar relaciones habituales con ciudadanos del país que les acoge, puesto que el $100 \%$ de la muestra sostiene que tiene algún amigo o conocido español.

\subsection{La integración en España: una percepción positiva para los rusos}

Para los fines del proyecto general y de este estudio, resultaba relevante extraer la autopercepción sobre la integración del colectivo en España. El $45 \%$ de los rusos revela sentirse muy integrado y el $38 \%$ bastante; por lo que se constata que la inmensa mayoría afirma tener un alto grado de integración en España. A este respecto, en el grupo de discusión, además del idioma español como una barrera lingüística y cultural para algunos de sus compatriotas, también achacaban a la idiosincrasia de la población rusa como un elemento decisivo en la integración, sin olvidarse de enfatizar que los rusos con un nivel económico muy alto tienen pocas intenciones de integrarse, ya que «viven como en una burbuja» en sus urbanizaciones o chalets de lujo:

"Los españoles nos definen como muy fríos, como que no expresamos mucho nuestras emociones; entonces los rusos que expresan muchas emociones sí que se integran más rápido, pero luego otros rusos que son como el agua, son tranquilos y aquí no se entiende 
eso. Les tachan de arrogantes o que no tienen sangre. Pero aquí no siempre lo entiende la gente». (Mujer, operadora de logística, 7 años viviendo en España).

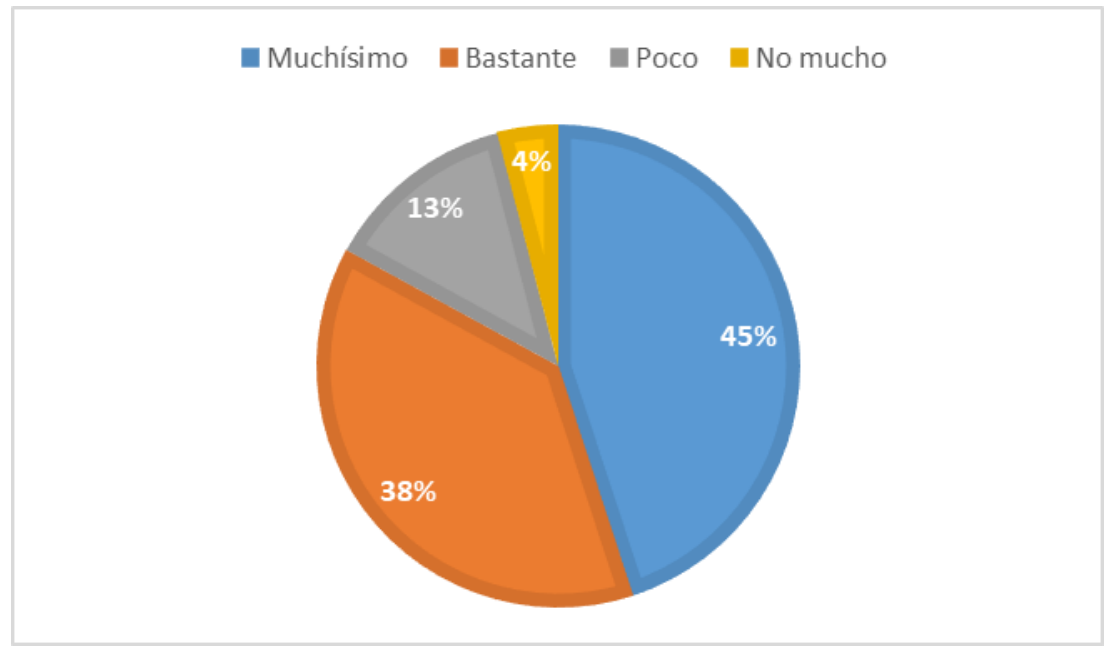

Figura 2. Resultados totales a la pregunta «¿Cómo de integrado se siente usted aquí?» Fuente: elaboración propia.

Otro signo evidente de integración de los rusos es el grado de conocimiento del lugar donde viven en la Costa del Sol y del estilo de vida español. Nuestra investigación arroja buenos resultados por parte de los ciudadanos rusos en ambos aspectos, por encima del $80 \%$. Por el contrario, baja al $60 \%$ el porcentaje de rusos que conoce bien o muy bien el estado actual de España en materia financiera, política y social.

\subsection{La utilidad social de los medios en ruso en la Costa del Sol}

En esta investigación también se abordó de manera más específica el papel que desempeñan los medios de comunicación destinados a los rusos como aspecto que ayuda a su integración en España, aspecto esencial de esta prensa, ya explorado, entre otros, por López Romero (2009b).

En primer lugar, destaca que un $75 \%$ de los rusos encuestados consume, de modo regular, medios de comunicación producidos en su propio país (prensa, radio, televisión o Internet). Como principales razones argumentan que se debe a que esos medios de comunicación les informan sobre Rusia (30\%) y a que les ayudan a sentirse cerca de su país de origen (30\%). Por tanto, se colige que, en buena medida, esos medios continúan siendo sus referentes emocionales, pese a vivir fuera de Rusia, lo que les permite estar al tanto de la actualidad de su país.

En el caso de los medios de comunicación producidos en la Costa del Sol para extranjeros, es decir, en idiomas diferentes al español, un $64 \%$ de rusos de nuestra muestra los consume. En concreto, los tres medios más consumidos por los rusos encuestados son tres periódicos (por orden, el primero y el tercero, en ruso; el segundo, en inglés): Mesto Vstrechi, Sur in English y Sur en ruso. 


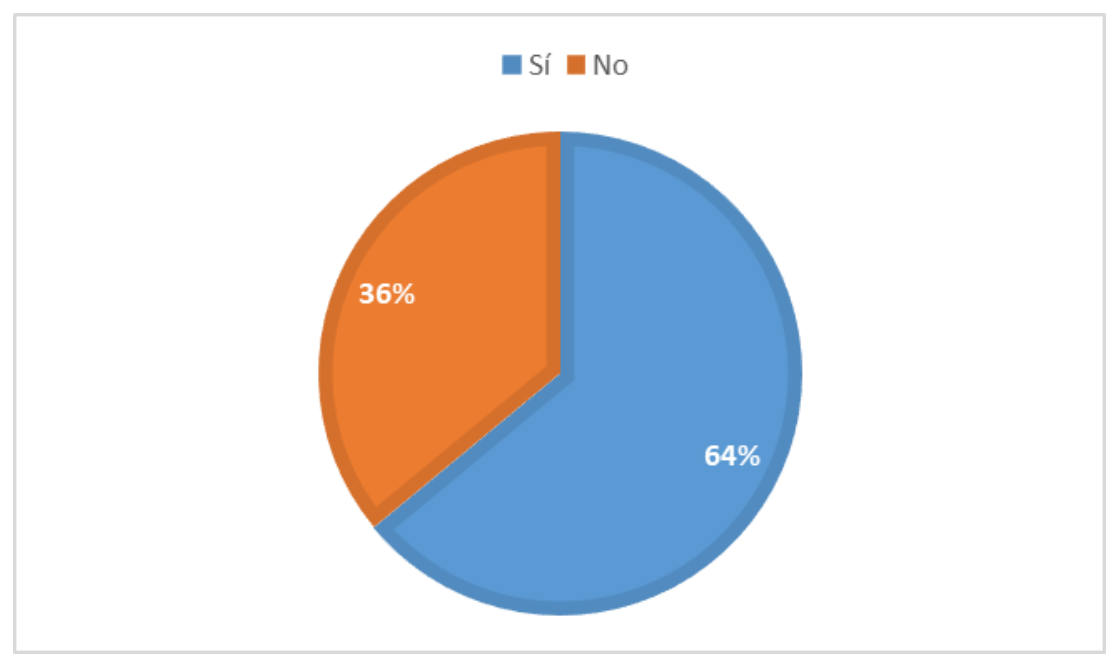

Figura 3. Resultados totales a la pregunta «¿Consume medios en un idioma diferente al español que se produzcan en la Costa del Sol?»

Fuente: elaboración propia.

Del análisis por separado de cada tipología de medios de comunicación y considerando si son consumidos siempre, eventualmente, a veces o casi nunca, los ciudadanos rusos usan más, de modo regular, las páginas web (30\%), la televisión (30\%) y la radio (29\%). A pesar de que el consumo es inferior para los periódicos $(11 \%)$, es paradójicamente con este medio de comunicación con el que los residentes rusos están más satisfechos (40\%), mientras que el grado de satisfacción más bajo se lo adjudica la televisión (31\%).

A la pregunta de cuáles eran las razones para consumir medios producidos en la Costa del Sol en idiomas distintos al español, los encuestados esgrimían, en primer lugar, que no entienden los medios españoles (29\%) y, en segundo lugar, respondían que están interesados en lo que pasa en la Costa del Sol (24\%). Por consiguiente, de estos datos se puede inferir que hay un cierto porcentaje de ciudadanos rusos que prefiere informarse de la actualidad de esta zona de España a través de medios elaborados en ruso o en otros idiomas en lugar de hacerlo en español. Sin embargo, según lo expresado en los grupos de discusión, aquellos residentes rusos que dominan ya el idioma prefieren los medios en español para estar más conectados al país que les acoge:

«Si pasa algo grave en Rusia sí leemos la prensa rusa. Pero si se trata de noticias internacionales o de lo que pasa en España obviamente yo leo la prensa española». (Mujer, promotora turística, 12 años viviendo en España)

En opinión de los encuestados rusos, las noticias más interesantes que ofrecen los medios para extranjeros en la Costa del Sol son las de cultura y tiempo libre (13\%), las relativas a España (12\%) y las de eventos locales (11\%). Por consiguiente, de estos medios les interesa predominantemente el periodismo de servicios, como así queda reflejado también en el alto porcentaje de rusos (89\%) que considera que dichos medios les aporta más conocimiento del territorio de acogida, en este caso, la Costa del Sol. Un 80\% manifesta que les ayuda a conocer 
mejor la sociedad española; por lo que a estos medios les confieren también un carácter divulgativo de cómo se constituye socialmente España en la actualidad.

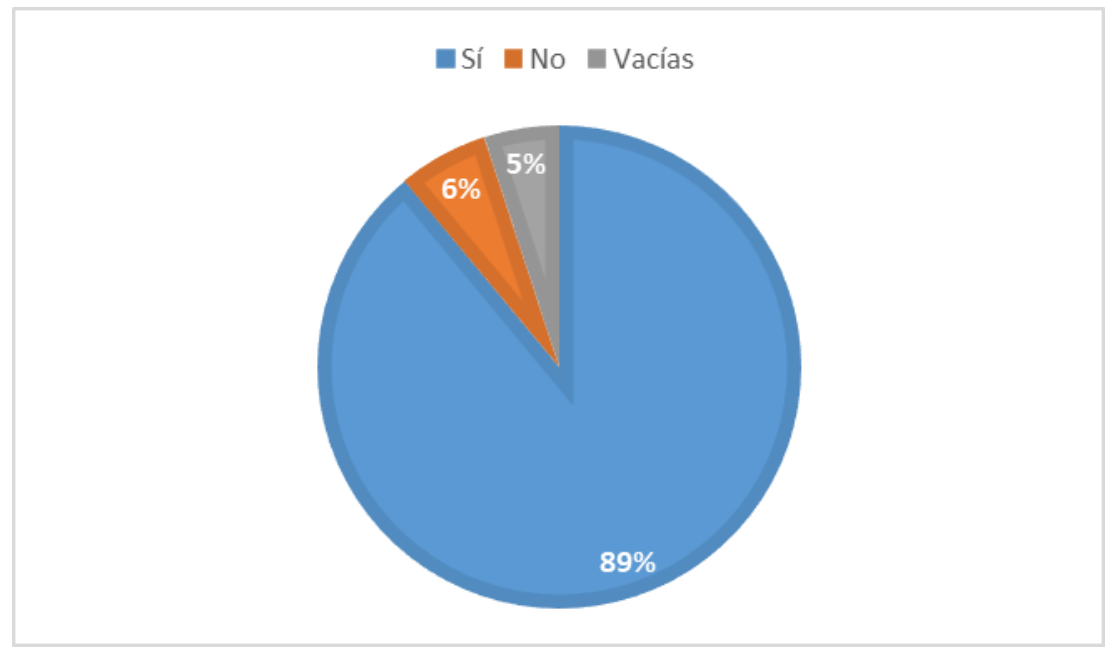

Figura 4. Resultados totales a la pregunta «¿Cree que estos medios le dan más conocimiento del sitio donde vive?»

Fuente: elaboración propia.

Como ya se ha indicado anteriormente, uno de los aspectos en el que más se pretendía profundizar en el estudio era en el proceso de integración en la sociedad española a través de los medios destinados a los residentes rusos. A tenor de los resultados, tienen una percepción bastante positiva del papel vertebrador de estos medios: en primera instancia, para unir mejor a los miembros de la comunidad rusa residente (un 73\% así lo piensa); y, en segundo término, para fomentar la integración entre la comunidad rusa y la sociedad española (un 69\% así lo apunta). Incluso más de la mitad de la muestra (56\%) piensa que estos medios les ayuda a aprender español. En cambio, son muchos menos (el $45 \%$ ) los que usan esos medios para seguir conectados con Rusia.

\subsection{Al servicio de la publicidad}

A pesar de que en los medios en lengua rusa elaborados en la Costa del Sol, en especial en las revistas, las páginas de publicidad superan a las de información (Pozo García, 2019), para el 56\% de los encuestados la publicidad en esos medios les aporta información útil. No obstante, en el grupo de discusión los participantes fueron muy críticos acerca de este contenido publicitario, ya que la comunidad rusa en la Costa del Sol es relativamente pequeña en comparación a otras comunidades, por lo que afirmaron que les funciona principalmente el boca a boca.

En definitiva, en el grupo de discusión hicieron hincapié en que los residentes de Rusia con menos interés en aprender el idioma español y, en consecuencia, integrarse en la sociedad española, son los rusos más acaudalados, que llegaron para instalarse en urbanizaciones de lujo de Marbella, Benahavis y Sotogrande; de ahí que los participantes en el grupo de discusión conciban a los medios elaborados en ruso como instrumentos de comercialización de productos de alta gama (joyas, relojes, belleza, cosmética, vehículos, etc.) y de servicios para los residentes rusos con mayor poder adquisitivo (despachos de abogados, inmobiliarias, clínicas privadas, 
etc.). Hay que señalar que en su mayoría se trata de medios gratuitos cuya base primordial de ingresos es la publicidad.

"Las personas ricas que viven en Marbella no tienen motivos para integrarse en esta sociedad, porque no están aquí para trabajar, no les hace falta, no tienen motivos para aprender castellano y por eso no pueden aprender bien castellano y de eso se aprovechan los medios en ruso» (Hombre, guía turístico, 18 años viviendo en España).

\section{CONCLUSIONES}

La oferta mediática local para la comunidad rusa en la Costa del Sol se compone mayoritariamente de medios escritos, en un número bastante considerable para la cantidad de residentes de esta nacionalidad en la zona. No obstante, habría que recalcar que el público objetivo de los medios de comunicación en lengua rusa en España en general y en la Costa del Sol en particular es mucho más amplio, puesto que se dirige también a otras nacionalidades rusohablantes: ucranianos, bielorrusos, kazajos, armenios, etc.

El principal formato son las revistas, que se caracterizan por su atención a informaciones de estilo de vida y su cuidado diseño y que cumplen también una función publicitaria muy evidente. En parte, ello guarda relación con un perfil de residente ruso de alto poder adquisitivo, radicado prioritariamente en Marbella, Benahavis y Sotogrande, pero que debemos decir que se ve completado con el de los trabajadores de clase media -o mejor dicho trabajadoras, dado el carácter eminentemente femenino de la emigración rusa en el territorio- bastante jóvenes (sobre todo en comparación con otras poblaciones de residentes mayoritariamente jubilados como la británica o la alemana). Otros rasgos predominantes son la intención por el momento de permanecer en la Costa del Sol, con un tiempo de estancia ya largo e incluso la presencia de red familiar, mostrándose satisfechos con la calidad de vida en su actual lugar de residencia.

Así pues, en cuanto a la integración encontramos quizás que el dominio del español resulta una pista clave del perfil, repartido entre quienes por trabajar con otros ciudadanos rusos o por su alto nivel de vida no necesitan apenas manejarse en español y los que por relaciones laborales o familiares con españoles dominan la lengua. A su vez, este mayor o menor dominio del español está relacionado con la necesidad de consumir medios locales en ruso, que pueden hacerse más imprescindibles en los momentos iniciales de la estancia.

En cuanto a la vinculación con otros ciudadanos rusos residentes, coexisten las organizaciones en las que este colectivo se asocia y se relaciona de manera presencial con una importante penetración de las redes sociales online.

Pese a preferir mayoritariamente informarse a través de los medios de su país de origen, un $64 \%$ de rusos de nuestra muestra afirma consumir medios de comunicación producidos en la Costa del Sol para extranjeros. Resulta interesante que, pese a existir una oferta atractiva de revistas, así como dos emisoras de radio, los tres medios más consumidos por los rusos encuestados son tres periódicos, uno de ellos en inglés, lo que da muestra, por un lado, del tipo de informaciones 
prácticas y de actualidad que interesan mayoritariamente, y, por otro, del carácter cosmopolita de este colectivo y de la zona en la que se inserta.

Asimismo, el $89 \%$ de los encuestados considera que dichos medios locales en ruso les aportan un mayor conocimiento del territorio de destino y un $80 \%$ manifiesta que les ayuda a conocer mejor la sociedad española. Todo ello nos lleva a colegir la necesidad específica de un pueblo geográfica y culturalmente más alejado del español de entender, mediante estos medios, el carácter y rasgos identitarios del área de acogida, ya que muchos enfatizan las diferencias y las dificultades de comprensión mutua. Así pues, en términos generales la percepción del papel vertebrador de estos medios resulta bastante positiva.

No obstante, los medios de comunicación en lengua rusa en la Costa del Sol no quedan exentos de crítica por parte de sus públicos, sobre todo la derivada de la descompensación entre publicidad e información en algunos de estos medios. Ello constituye un ejemplo de la sutil línea entre una oferta mediática útil para un segmento de la población, con información de actualidad y servicios, y unos soportes entendidos como nichos de negocio. Ejemplos de ambos modelos se dan en los medios dirigidos a la población rusa, a veces conviviendo incluso en la misma publicación. En cualquier caso, también es cierto que, como hemos podido comprobar, este colectivo experimenta una dualidad muy fuerte entre la población trabajadora y el núcleo de residentes de alto poder adquisitivo, lo cual se trasluce también en los medios locales destinados a residentes rusos en la Costa del Sol.

\section{Referencias}

Barrera-Fernández, D. (2015). The Russian real estate market in Spain. Architecture on the Costa del Sol. 5th All-Russian research and practice conference with international participation: «Investments and real estate as a material basis for economy modernization and innovation».

Byford, A. (2012). "The Russian diaspora in international relations: "Compatriots» in Britain". Europe-Asia Studies, 64 (4), pág. 715-735. DOI: https://doi.org/10.1080/09668136.2012.660764

Ershov, V. F. \& Gadzhieva, K. K. (2018). Russian community in Spain and global cultural dialogue (19912015). RUDN Journal of Russian History, 17 (1), pág. 92-108. DOI: http://dx.doi.org/10.22363/2312-8674-2018-17-1-92-108

García Galindo, J. A. (1998). El papel de los medios de comunicación en la configuración y promoción de la Costa del Sol. En VV. AA. (Ed.), Historia de La Costa del Sol. Diario Sur: Málaga.

García Galindo, J. A. (2004). Prensa y turismo en España (Málaga, 1872-1936). En N. Ludec y F. Dubosquet (coords.), Centros y periferias: prensa, impresos y territorios en el mundo hispánico contemporáneo: homenaje a Jacqueline Covo-Maurice (pp. 169-178). Pessac: Université Michel de Montaigne-Bordeaux 3/PILAR.

García Galindo, J. A. y López, L. (2018). Los medios en lengua extranjera. Diversidad Cultural e integración. Granada: Comares. 
García Galindo, J. A. y Cuartero, A. (2019). "Los medios de los residentes comunitarios en el proceso de integración europea: otra forma de globalización mediática”. En: Rêgo, Ana Regina, et al. Os desafios da pesquisa em história da comunicação: entre a historicidade e as lacunas da historiografia. Brasil, Porto Alegre: EDIPUCRS Editora Universitária da PUCRS

García Galindo, J. A.; Novas Martín, G. y López Romero, L. (2020): “La prensa en inglés en la Costa del Sol: roles, funciones y perfil de los lectores" Estudios sobre el Mensaje Periodístico 26 (3), 951-962.

Harmon-Donovan, D. J. (2015). A history of the Soviet diaspora in the post-cold war era (1989-2015): Case studies of Russian-speakers in Souh Korea, Cambodia and The Netherlands. Tesis doctoral. Texas State University, San Marcos, Texas.

Lario Bastida, M. (2008). Medios para minorías y minorías en los medios. Inmigración, discurso y medios de comunicación, 65.

Larrubia, R. y Ocaña, M. C. (2014). Extranjeros en Andalucía, diversificación de las corrientes inmigratorias y sus patrones de distribución sobre el espacio andaluz. Ería. Revista Cuatrimestral de Geografía, 93(2014), pág. 101-119.

López Romero, L. (2009). Prensa y comunidad extranjera en España. Estudio descriptivo y analítico del caso malagueño. Madrid: Quiasmo Editorial.

López Romero, Laura (2009b). "Prensa extranjera en España, la integración social a través de los medios escritos". Telos: Cuadernos de comunicación e innovación, 80: 116-123.

Lunt, P. \& Livingstone, S. (1996). Rethinking the Focus Group in Media and Communications Research. Journal of Communication, vol. 46 (2), pág. 79-98. DOI: https://doi.org/10.1111/j.14602466.1996.tb01475.x

Martínez Pastor, E., \& Santín Durán, M. (2009). Publicaciones para inmigrantes: Estudio de las informaciones y la publicidad dirigidas a la comunidad inmigrante. Doxa comunicación, 9, pág. 125-144. DOI: 10.31921/doxacom.

Perotto, M. (2014). Post-Soviet Russian-speaking diaspora in Italy. In L. Ryazanova-Clarke, (Ed.), The Russian Language Outside the Nation. Edinburgh University Press, pág. 143-165.

Pivovarov, E. G. (2015). Historiography of the Russian diaspora in the USA. Социология науки и технологий, 6 (1), pág. 113-121.

Pozo García, J. C. (2019). El panorama mediático en lengua rusa en la Costa del Sol. En J. A. García Galindo, y L. López Romero, (eds.), Los medios en lengua extranjera: Diversidad cultural e integración. Granada: Editorial Comares, pág. 33-41.

Prokopenko, Y. (2014). Evolución del flujo turístico ruso y su importancia económica: el caso de la Costa Blanca. Tesis doctoral. Instituto Universitario de Investigaciones Turísticas, Universidad de Alicante.

Shovgenin, A. N. \& Shovgenina, E. A. (2015). The newspapers of Russian-speaking diaspora in Germany and its sociolinguistic space. Russian Linguistic Bulletin, pág. 17-21. DOI:10.18454/RULB.4.10 
Suárez-Ortega, M (2005). El grupo de discusión. Una herramienta para la investigación cualitativa. Barcelona: Laertes.

Tereshchuk, A. (2017). La asimilación lingüística de los inmigrantes rusos en Barcelona. Tesis doctoral. Universidad de Barcelona.

\section{Notas}

${ }^{1}$ Este artículo forma parte de los resultados del Proyecto I+D "Medios de comunicación y construcción europea: estudio sociocomunicativo de los residentes comunitarios en el sur de España y de Portugal" (2016-2019), dirigido por el catedrático de Periodismo Juan Antonio García Galindo. (cf. www.transfomedia.uma.es).

${ }^{2}$ La traducción del inglés al español es nuestra.

${ }^{3}$ La traducción desde el ruso es nuestra de aquí en adelante.

${ }^{4}$ INE. Revisión del padrón de habitantes. 2019.

${ }^{5}$ INE. Revisión del padrón de habitantes. 2018.

${ }^{6}$ Instituto de Estadística y Cartografía de Andalucía. Padrón Municipal de Habitantes. 2017. 